\title{
L'influence de la tutelle mandataire française sur l'identification des élites syriennes et libanaises devant la société argentine (1900-1946)
}

The Influence of the French Mandate on the Identification of the Syrian and Lebanese Elites in front of the Argentinian Society (1900-1946)

Influencia de la tutela del mandato francés en la identificación de las elites sirias y libanesas frente a la sociedad argentina (1900-1946)

\section{Gildas Brégain}

\section{OpenEdition}

\section{Journals}

Édition électronique

URL : https://journals.openedition.org/remi/5598

DOI : $10.4000 /$ remi.5598

ISSN : $1777-5418$

Éditeur

Université de Poitiers

Édition imprimée

Date de publication : 1 décembre 2011

Pagination : 177-199

ISBN : 979-10-90426-02-3

ISSN : 0765-0752

\section{Référence électronique}

Gildas Brégain, «L'influence de la tutelle mandataire française sur l'identification des élites syriennes et libanaises devant la société argentine (1900-1946) », Revue européenne des migrations internationales [En ligne], vol. $27-n^{\circ} 3$ | 2011, mis en ligne le 01 décembre 2014, consulté le 15 avril 2022. URL : http://journals.openedition.org/remi/5598; DOI : https://doi.org/10.4000/remi.5598 


\section{Note de recherche}

\section{L'influence de la tutelle mandataire française sur l'identification des élites syriennes et libanaises devant la société argentine (1900-1946)}

\section{Gildas BRÉGAIN ${ }^{1}$}

《 Turco de mierda », « Turco bruto »... telles sont les insultes que plusieurs l'autre, lors de leur séjour en Amérique latine. Au début de la Première Guerre mondiale, plus de 100000 Ottomans sont présents en Argentine, exerçant pour la plupart le métier de colporteurs et de petits marchands. Dès la fin du XIXe siècle, les dirigeants argentins considèrent que ces immigrants qui se consacrent uniquement au commerce sont inutiles au développement économique du pays. De langue arabe, principalement originaires de la province de Syrie et plus particulièrement du Mont-Liban, ces Ottomans sont bien loin du modèle de l'immigrant européen agriculteur. Juan Alsina, directeur du Département d'Immigration de 1891 à 1910 , dénonce ainsi le caractère « exotique » du Turco ${ }^{2}$, potentiellement nuisible à la construction du crisol de razas argentin. La société argentine attribue de façon simplificatrice à l'immigrant issu du Moyen-Orient et de langue arabe le surnom de Turco, qu'il soit chrétien, musulman ou juif. Elle construit ce stéréotype largement péjoratif, non seulement sur son appartenance à la religion musulmane, mais également sur l'étrangeté de ses coutumes, son improductivité et son analphabétisme (Bestene, 1994). Cette catégorisation d'origine populaire s'explique notamment par l'influence d'une culture européenne qui affuble de « turcs » ou « turcos » tous ceux qu'elle perçoit comme musulmans depuis le XIIe siècle ${ }^{3}$.

L'attitude discriminatoire des populations à leur égard n'empêche pas les immigrés syriens et libanais de pénétrer rapidement l'économie argentine et de s'insérer

1 Doctorant en histoire contemporaine, CNRS - UMR 6258 CERHIO, Université de Rennes II ; gildasbregain@hotmail.fr

2 Memorias del Departamento General de Inmigración, Buenos Aires, 1899, p. 84.

3À la fin du XIXe siècle, le terme de turcos est couramment utilisé en France pour désigner les tirailleurs algériens. Pellerin, lithographie intitulée Tirailleurs algériens ou turcos, 1864. 
dans l'industrie, les professions libérales et la politique à partir des années 1920 . Néanmoins, les plus fortunés d'entre eux éprouvent des difficultés à intégrer la haute société. C'est pourquoi, afin d'améliorer leur image, une trentaine d'élites syriennes et libanaises prennent la défense de leurs compatriotes face à la nation argentine. Elles contestent le qualificatif de Turcos au profit de nouvelles identifications valorisantes. La plupart de ces personnalités sont des Maronites originaires du Mont-Liban : parmi eux, des intellectuels écrivent régulièrement des articles dans la presse en langue arabe (Rachid Rustom, Habib Estefano, Alejandro Schamun, Checri Abi Saab, Esbir Gorayeb, José Juairy, Jorge Assef, Antonio Arida, Massoud Posleman), d'autres sont des religieux (père Elias M. Gorayeb, père Miguel Latuf Inderi). On y trouve aussi des commerçants (Moisés et Salomon Busader, Domingo Kairuz, Miguel Samra, Salomon David Gebara). Jorge Sawaya, Elias Amar, Khalil Sa'adeh sont des intellectuels libanais de religion orthodoxe, tandis que l'intellectuel Nagib Baaclini est un melkite. L'Émir Emin Arslan, qui occupe la fonction prestigieuse de consul ottoman à Buenos Aires de 1910 à 1915, et Ta'an Felipe Homad, sont des Libanais druses. Enfin, Ibrahim Hussein Hallar et Mohamed Murad sont des Libanais musulmans, et Constantin Melhem est un ex-orthodoxe de Tripoli, converti ensuite à l'islamisme. Plusieurs élites sont des Syriens orthodoxes, qu'ils soient rédacteurs de journaux (Husny Abdul Malik, Miguel Cosma), religieux (Monseigneur Mikael Jaluf, archiprêtre Moise Hillar) ou commerçants (Moisés José Azize, Mecia Dantur). José Jorge est un commerçant syrien de religion juive. Enfin, Saiffudin Rahhal est un musulman égyptien. Certains d'entre eux correspondent régulièrement avec les leaders nationalistes du Moyen-Orient, du fait de leur parenté, tel l'Émir Emin Arslan, cousin de Chekib Arslan, et Nagib Baaclini, qui a un oncle Prélat à Beyrouth, Pedro Baaclini. Jusqu'à la fin de la Première Guerre mondiale, les élites ottomanes de Buenos Aires ont des contacts fréquents avec celles de Montevideo, ce qui justifie l'étude des identifications construites par leurs compatriotes de Montevideo à cette période.

Pour légitimer l'intégration de leurs compatriotes au sein de la société argentine, les élites sont tenues de présenter l'image d'une seule et même collectivité, issue d'une seule « race » ou d'un conglomérat de « races », afin de pouvoir prétendre enrichir la nation d'accueil culturellement, économiquement, etc. Dans ce cadre, les élites doivent absolument tenir compte de la composition contemporaine des collectivités syriennes et libanaises présentes en Argentine. Si les Ottomans maronites originaires de la montagne libanaise sont majoritaires au début du XXe siècle, à la fin des années 1920 les Syriens sont désormais supérieurs en nombre, et Musulmans et Orthodoxes semblent aussi nombreux que les Maronites. Les autres immigrants de langue arabe (Palestiniens, Irakiens, etc.) sont très peu nombreux.

Les nouvelles identifications construites par ces élites sont influencées à divers degrés par les catégorisations opérées par la société argentine, l'administration argentine et les agences consulaires et diplomatiques françaises. Contrairement à beaucoup de pays américains, les autorités argentines n'ont pas mis en place de législation interdisant l'immigration des «Asiatiques ». De 1880 à 1946, l'administration argentine catégorise ces immigrés sous des vocables différents : otomanos, turcos, asiáticos, árabes, sirios, libaneses, palestinos. Ces dénominations exercent peu d'influence sur le processus d'identification des élites syriennes et libanaises devant la société argentine, si on les compare aux identifications imposées par les agences consulaires et diplomatiques françaises. 
À la fin de la Première Guerre mondiale, l'Empire ottoman se retrouve disloqué et en dépit de la volonté populaire, les puissances européennes choisissent de diviser les provinces arabes en différents États. Le $1^{\text {er }}$ septembre 1920, la France déclare l'indépendance du Grand Liban vis-à-vis de la Syrie. En 1923, la Société des Nations charge officiellement la France d'un Mandat sur la Syrie et le Liban, autorisant ainsi ses agences consulaires à délivrer des papiers d'identité aux émigrés syriens et libanais. En réalité, dès les premières années du XXe siècle, ces derniers sollicitent déjà les autorités françaises en Amérique.

Jusqu'au début de la Première Guerre mondiale, la plupart des élites ottomanes présentes en Argentine se voient comme des sujets ottomans, servant un Empire multinational, multiethnique et multireligieux. Elles refusent toutefois le qualificatif de Turco, car il est utilisé péjorativement, à la fois dans le pays d'accueil, et à la cour ottomane, où il désigne les paysans analphabètes et un peu naïfs d'Anatolie dans les histoires humoristiques. Face à la société argentine, la plupart des élites se présentent comme otomanos et/ou sirios, puisque la Grande Syrie constitue alors une province autonome au sein de l'Empire ottoman. Néanmoins, leur identification diffère sensiblement selon le contexte et l'interlocuteur auquel elles s'adressent (Cooper, 2005 : 71). Ainsi, en privé, lors d'un dialogue avec l'un de ses compatriotes, un dirigeant va plutôt s'identifier par son village d'origine et son appartenance religieuse. Lorsqu'elles entrent officiellement en contact avec les autorités françaises, certaines élites maronites mettent en valeur leur proximité culturelle avec la France, et s'identifient comme Libanais. Ainsi, Checri Abi Saab prend sa plume aux côtés d'Alejandro Safi au nom des « Maronites du Liban » pour présenter ses vœux au ministre de la République française à Montevideo à l'occasion du 14 juillet $1905^{4}$. Certains responsables ont alors conscience de leur identité libanaise, qu'ils conçoivent comme un complément à l'identité syrienne ou ottomane/syrienne, mais ils n'éprouvent pas le besoin de la mettre en relief lorsqu'ils s'adressent à la société d'accueil.

Dès les premières années du XXe siècle, les autorités françaises influencent de multiples manières les identifications que les élites promeuvent face à la société argentine.

\section{LA FRANCE STRUCTURE LES IDENTIFICATIONS DES ÉLITES OTTOMANES ÉMIGRÉES (1900-1923)}

Depuis la fin du XIXe siècle, la France a des visées coloniales sur le territoire de la Grande Syrie. Afin de soutenir sa politique d'implantation au Levant, elle manipule les collectivités ottomanes émigrées, et structure fortement l'identification de leurs élites.

4 Archives du ministère français des Affaires étrangères de Nantes (AMAE-N), Montevideo (Mon), Car 46, lettre du 14/7/1905, San Fructuoso, Checri Abi Saab et Alejandro Safi au Min de la Rép. Frcse à Montevideo. 


\section{Les élites invoquent leur européanité pour légitimer leur argentinité (1900-1919)}

Dès le début du XXe siècle, les élites syriennes et libanaises font tout pour « argentiniser » leurs compatriotes, afin de faire oublier le caractère exotique que la société d'accueil leur attribue. Pour promouvoir l'assimilation de leurs compatriotes au milieu argentin, ces élites présentent l'image d'un immigrant idéal, sain de corps et d'esprit, travailleur acharné, et respectueux des lois. Certaines d'entre elles invoquent aussi leur européanité, car les Argentins apprécient les cultures européenne et française, à l'inverse de la culture arabe. Revendiquer leur culture leur permet de contester l'image du Turco analphabète. L'un de leurs amis, le député argentin Joaquín V González, devient d'ailleurs le porte-parole des élites ottomanes lors d'un débat parlementaire. En 1910, il défend l'immigration syrienne, très critiquée jusque-là, en déclarant que « les Syriens, en quelque sorte, sont les Européens de l'Asie, avec leur antique civilisation, leur morale et leur culture chrétienne. Ils sont placés sous la protection des puissances européennes, et pratiquent l'éducation, les langues et le progrès comme les Européens ${ }^{5}$. En développant un tel discours, les élites ottomanes se différencient des "Asiatiques » perçus négativement par les dirigeants argentins, et prétendent appartenir aux populations de culture européenne et catholique, bref, « civilisées ».

Jusqu'à la mise en place du Mandat français, la grande majorité de ces élites ottomanes est imprégnée de culture française et admire la France. Beaucoup ont appris le français et l'histoire mythique des liens entre la France et la Syrie dans les établissements scolaires des missionnaires français en Syrie. L'identification de ces élites à la France se perçoit nettement dans les cérémonies patriotiques. Par exemple, lors des fêtes en l'honneur de la restitution de la constitution ottomane en 1908, on déploie les drapeaux argentin, ottoman et français et on joue l'hymne de chacun de ces pays. Cas exceptionnel, Jorge Sawaya a reçu une éducation à l'université américaine de Beyrouth et s'écarte de l'influence culturelle française.

Tout au long du XXe siècle, les élites originaires de la Grande Syrie s'efforcent de construire une identification argentine pour elles et leurs compatriotes. Toutefois, à partir de 1919, lorsque les prétentions coloniales de la France sur la Grande Syrie apparaissent trop manifestement, un grand nombre d'entre elles (Salomon Busader, Moisés Busader, Alejandro Schamun, etc.) cessent de s'identifier à la France. De ce fait, elles n'utilisent plus la référence française ou européenne pour légitimer leur argentinité. Celles qui sont favorables à une Grande Syrie arabe (Khalil Sa'adeh, Jorge Sawaya, Ta'an Homad) s'opposent violemment à toute identification à la France et refusent que soit hissé un drapeau libanais ou syrien comportant un modèle réduit du drapeau français, symbole du Mandat. L'Émir druse Emin Arslan, en tant que directeur de la Revista de Francia, continue de louer l'apport « civilisateur » de la France au Levant jusqu'en 1923, moment où il commence à critiquer le Mandat français. Ce sont surtout les missionnaires maronites (père Elias $\mathrm{M}$. Gorayeb, père Miguel Latuf Inderi), ainsi que certains intellectuels maronites groupés au sein de l'Alliance Libanaise (José Juairy, Antonio Arida, Checri Abi Saab) qui continuent

5 Diario de sesiones de la Cámara de senadores de Argentina, Buenos Aires (BA), 12 de septiembre de 1910, p. 558. 
à construire cette identification à la France pour légitimer leur argentinité. Les religieux maronites se présentent alors comme les « petits Français de L'Orient » et les drapeaux argentins, libanais et français sont arborés à chaque cérémonie patriotique dans le Colegio San Marón de Buenos Aires ${ }^{6}$.

\section{Les agents français soutiennent les identifications sirianos et libanenses}

Le travail de catégorisation réalisé par les autorités consulaires françaises, lorsqu'elles remettent des papiers d'identité, affecte de façon sensible l'identification des intellectuels ottomans. À partir de 1903, bien que ces émigrés aient la nationalité ottomane, les agents consulaires français en Uruguay délivrent aux Chrétiens ottomans des papiers d'identité attestant de leur nacionalidad syriana. Les fonctionnaires le font de leur propre initiative ou à la demande des intéressés. La terminologie utilisée sur les papiers d'identité peut s'expliquer soit par la translittération du français «syrienne » en syriana, soit par la reprise du latin syriana. Les élites intériorisent alors les caractéristiques de cette attribution administrative et se proclament sirianos devant la société d'accueil, fondant en 1906 la Sociedad Siriana à Montevideo. Cela ne les empêche pas de se reconnaître aussi comme Ottomans. Ainsi, ayant reçu en 1903 du consul de France à Montevideo un certificat de nacionalidad syriana, Checri Abi Saab rédige en tant que membre de la Sociedad Siriana un discours louant la restauration de la Constitution de Turquie en juillet $1908^{7}$. Au contraire, en Argentine, la plupart des intellectuels ne se définissent pas comme sirianos, mais comme sirios et/ou comme otomanos (Jozami, 1993 : 194-195). Ainsi, en 1902, les missionnaires maronites fondent le Colegio Sirio-argentino à Buenos Aires.

À partir de 1915, la France soutient certains dirigeants soucieux d'affirmer leur identité libanaise, en autorisant la remise de certificats de nationalité libanense. Toutefois, la présence d'une association Juventud Libanense à Montevideo en juillet $1908^{8}$ prouve l'existence dès cette époque d'une identification libanaise indépendante de la catégorisation administrative française. Les jeunes intellectuels maronites fondateurs de cette association reprennent l'identification libanense, un terme d'origine latine, mais déjà couramment utilisé en espagnol pour désigner la montagne libanaise. Amorcée dans les années 1840 par le clergé maronite, la construction de l'identification libanaise est consolidée à partir de 1861 par la constitution du Mont-Liban en Mutasarrifiyya, région autonome au sein de la province de Syrie. Cependant, jusqu'au début du XXe siècle, cette identité libanaise n'est pas conçue comme antagonique à l'Empire ottoman, ni à la Syrie. Ainsi, à Montevideo, la Juventud Libanense participe à la cérémonie en l'honneur de la constitution ottomane en 1908.

Après cette date, face à la politique de centralisation mise en œuvre par les Jeunes Turcs, plusieurs intellectuels originaires du Mont-Liban veulent sauvegarder l'autonomie de leur région. C'est pourquoi certains d'entre eux, émigrés en Égypte, créent en 1909

6 El Misionero, BA, diciembre $1^{\text {er }}$ de 1928.

7 AMAE-N, Mon, Car 241, art. La Constitución de Turquía. Una fiesta de la colonia, journal non spécifié, 24/8/1908.

8 AMAE-N, Mon, Car 241, carta, julio 14 de 1908, Montevideo, la Juventud Libanense al Señor Ministro de la Republica Francesa en el Uruguay. 
l'Alliance Libanaise, qui revendique l'indépendance absolue du Liban dans ses frontières « naturelles » (celles du Liban de Fakhreddine II) avec l'aide des Européens. Afin de légitimer cette revendication d'un Liban indépendant de l'Empire ottoman et de la Syrie, l'Alliance Libanaise invoque un passé mythique phénicien qui rapproche le Liban de l'Occident. Elle considère que le peuple libanais est le descendant des Phéniciens, un peuple qui appartenait à la " race sémitique », qui vivait du XIIe siècle au IVe siècle avant notre ère et avait colonisé le pourtour méditerranéen. Elle adopte comme emblème le cèdre du Liban. Ce groupe influence rapidement un cercle restreint d'intellectuels chrétiens à Montevideo et à Buenos Aires. Dans la capitale argentine, plusieurs élites originaires du Mont-Liban (dont Salomon David, Moisés et Salomon Busader) s'associent en mars 1915 pour fonder la Unión Libanense, afin de soutenir les Alliés - surtout la France - et de réclamer l'indépendance du Liban dans ses frontières « naturelles ». Puis, en juillet 1915, de l'autre côté du Rio de la Plata, à Montevideo, des certificats de nacionalidad libanense sont remis à certains émigrés par des associations soutenues par la Légation de France. Et, le 14 juillet 1917, grâce au soutien de l'ambassade de France, la Unión Libanense de Buenos Aires obtient pour la première fois l'autorisation d'arborer le drapeau libanais, orné d'un cèdre. Pourtant, la même année, cette association exprime des réticences lorsque le Dr Lakah et Mardam Bey veulent recruter pour la Légion d'Orient « sans distinction de race $"{ }^{9}$, car la Unión Libanense considère que les Libanais, les Syriens et les Arméniens ont des origines ethniques différentes.

À l'image des membres de l'Alliance Libanaise du Caire, les membres de l'Unión Libanense de Buenos Aires revendiquent leur origine phénicienne pour se différencier de la « race » et de la culture ottomanes. Au début du XXe siècle, ce phénicianisme est beaucoup plus développé dans les terres américaines et en Égypte que dans la province de Syrie elle-même (Kaufman, 2004 : 74). Depuis le milieu du XIXe siècle, les recherches françaises sur la période pré-islamique de la Grande Syrie s'intensifient et construisent un passé antique mythique pour cette région. La France encourage alors l'identification des Maronites du Liban aux Phéniciens, afin de s'attacher la collaboration de ce groupe ethnique minoritaire dans une optique colonialiste (Kaufman, 2004). Lorsqu'ils invoquent leur origine phénicienne pour démontrer leurs capacités civilisatrices à la société argentine, les dirigeants de l'Unión Libanense s'approprient donc une pratique coloniale française. Ceux-ci citent régulièrement les nombreuses colonies fondées par les Phéniciens sur le pourtour méditerranéen (comme Sidon, Tyr et Carthage), afin d'établir le rôle fondamental des Phéniciens dans la formation de la civilisation grecque. Checri Abi Saab écrit ainsi, en 1918, que les Maronites descendent « des anciens Phéniciens célèbres pour leurs audacieux voyages et leur commerce et auxquels on attribue l'invention de l'alphabet $»^{10}$.

Avant 1919, la théorie du phénicianisme n'est toutefois pas encore fixée : certains considèrent que l'ensemble des Syriens descendent des Phéniciens, tandis que d'autres estiment que seuls les Maronites du Mont-Liban ont ce privilège. Enfin, d'autres défendent l'idée que cette identité ethnique phénicienne s'est trouvée mélangée au cours des siècles à d'autres « races » du fait des invasions successives. En 1917, Alejandro Schamun se réfère ainsi aux qualités du conglomérat de « races » présent en Syrie : "Le Syrien tient du Phénicien l'instinct d'entrepreneur et la vaillance, qui est régulé par sa prédisposition

9 AMAE-N, BA (1880-1922), Car 102, Note sur l'Union Libanaise.

10 Abi Saab, Au Liban, Le Courrier de la Plata, BA, 14 juillet 1918.

REMI 2011 (27) 3 pp. 177-199 
au calcul ; de l'Égyptien antique, la sérénité d'esprit et l'amour de l'ordre ; du Grec, l'idéalisme libre et désintéressé, et de l'Arabe, l'imagination puissante et la candeur du sentiment » (Schamun, 1917 : 21). À l'image de la majorité des élites maronites présentes dans la grande province de Syrie, Alejandro Schamun est favorable à la constitution d'une Grande Syrie, avec une autonomie importante pour le Mont-Liban.

Jusqu'en 1920, le clergé maronite en Argentine refuse encore de s’identifier publiquement aux Phéniciens, car ceux-ci étaient païens. Mais en août 1919, le patriarche maronite Elias Hoyek arrive à Paris à la tête d'une délégation maronite, afin d'exiger l'établissement d'un Grand Liban à la conférence de la paix. Pour légitimer l'existence d'une communauté nationale distincte, fondée sur une différence ethnique et culturelle avec les Arabes, il invoque l'origine phénicienne des Libanais, ce qu'il n'avait jamais fait auparavant devant ses compatriotes maronites (Kaufman, 2004 : 86). Les revendications des religieux maronites en faveur d'un Grand Liban sont désormais liées à l'évocation d'un passé phénicien, y compris en Argentine. Lors d'une conférence publique, le père Elias Gorayeb déclare en 1927 que la nation libanaise est née depuis Saint Maron, et que les Libanais descendent des Phéniciens ${ }^{11}$.

\section{Les autorités françaises, créatrices de l'identification syro-libanaise}

Alors que cette identification libanaise commence tout juste à se consolider en une identité partagée dans les terres américaines, la France contribue à son affaiblissement à partir de la fin de l'année 1916. Elle encourage en effet la réunion des émigrés syriens et libanais au sein des mêmes associations afin de soutenir sa politique d'implantation au Levant. Dès la fin du mois d'octobre 1916, la Légation de France à Buenos Aires soutient la création de la Unión Siria pro-Independencia de Siria Libano, qui demande « l'indépendance de la Syrie et du Liban sous le Mandat de la France » ${ }^{12}$. L'Émir Emin Arslan, ancien consul ottoman à Buenos Aires, tient vite les rênes de l'association. Depuis Paris, Chekri Ganem, dirigeant du Comité Central Syrien, entre en contact avec lui à l'été 1917 pour le prier de procéder aux recrutements en faveur de la Légion d'Orient, sans succès. Quelques mois plus tard, le Comité Central Syrien envoie une mission de propagande en Amérique du Sud. Cette mission est chargée d'organiser le recrutement pour la Légion d'Orient, et de fusionner les collectivités syriennes et libanaises afin de soutenir les objectifs politiques français : la constitution d'une Grande Syrie fédérative, non arabe, sous l'égide de la France. Dans toutes les villes qu'elle traverse, cette mission encourage pour cela la fondation d'associations qualifiées de « syro-libanaise » ou « libano-syrienne ».

À Montevideo, où les immigrés sont quasiment tous originaires du MontLiban, et où préexiste une association El Despertar del Libano, Alejandro Safi forme un Comité Patriótico Libano-Siriano en juillet 1917, en prévision du passage de la mission. L'association a pour but de soutenir l'indépendance du Liban et de la Syrie sous protectorat de la France, et d'envoyer des volontaires pour défendre la patrie naissante. Mais en Argentine, la mission ne rencontre aucun succès, car la plupart des membres de la

11 Informe de la Asociación Protectora del Colegio San Marón, op. cit., pp. 53-54.

12 AMAE-N, BA (1880-1922), Car 102, lettre de la Unión Siria Pro Independencia de Siria Líbano, BA, le 13 sept 1920 à SE Mr Jean Roger Clausse, Min de France à BA. 
Unión Libanense de Buenos Aires, dirigée par le Maronite Salomon Busader, refusent de participer au recrutement pour la Légion d'Orient. Ils protestent ainsi contre le fait qu'on les confonde avec les Syriens ou d'autres peuples d'Asie Mineure ${ }^{13}$. Toutefois, certains Libanais, tels Checri Abi Saab et Alejandro Schamun, soutiennent la Unión Siria proIndependencia de Siria Libano. À partir de 1919, cette association tombe en désuétude, $\mathrm{du}$ fait que certains intellectuels libanais adoptent la position du patriarche maronite et se rallient à l'idée d'un Grand Liban. Checri Abi Saab quitte alors la Unión Siria pour intégrer L'Alliance Libanaise. Cette situation de déliquescence s'accentue en 1920, lorsque l'option Grande Syrienne s'identifie au gouvernement arabe de Faycal à Damas, ce qui pousse nombre de Libanais à rejeter l'option de la Grande Syrie fédérative.

Dirigée à la fin de l'année 1918 par Salomon David, la Unión Libanense connaît aussi de graves dissensions internes, puisque Salomon et Moisés Busader « affirment plus franchement leur opposition constante à la France ${ }^{14}$, conformément à l'évolution politique de l'Alliance Libanaise du Caire. La faction véritablement francophile des intellectuels libanais, menée par Salomon David, forme une nouvelle société paradoxalement appelée l'Alliance Libanaise, afin de soutenir le programme d'indépendance du Liban sous l'égide de la France. «Ce fut ainsi que se produisit le schisme au sein des collectivités libanaises $\rangle^{15}$. À la tête de la Unión Libanense, Moisés Busader et Massud Posleman prennent publiquement parti en faveur de l'indépendance complète de leur pays d'origine en 1920. Afin de défendre leurs idées auprès de la Ligue des Nations, ils envoient un plaidoyer au ministre des Affaires étrangères de la République argentine qui doit se rendre à Genève. Ils y affirment leur filiation avec « ce peuple, connu sous le nom de Phénicie » ${ }^{16}$, afin de montrer leur intelligence et leur capacité à s'autogouverner.

Le général Gouraud, Haut Commissaire de Syrie, consolide le processus de construction de l'identité nationale libanaise en créant le $1^{\text {er }}$ septembre 1920 un Grand Liban indépendant de la Syrie. Celui-ci englobe la plaine de la Bekaa et les villes côtières. Les Chrétiens, population majoritaire, détiennent le pouvoir. Cette proclamation de l'indépendance du Grand Liban est reçue avec satisfaction en Argentine par de nombreux intellectuels libanais, mais elle engendre certains questionnements personnels sur le contenu de l'identité libanaise : Checri Abi Saab évoque ainsi au Consul de France la difficulté de se réunir avec « les 15 mille [immigrés présents en Argentine] qui sont originaires des territoires qui ont été annexés au Vieux Liban pour en former le Grand Liban. Ceux-ci, qui ont été par-dessus le marché, étrangers à notre idiosyncrasie par le fait même d'avoir été régis par un régime tout à fait différent du nôtre, sont en leur majorité des musulmans, opposés par nature à nous $»^{17}$. Il déclare néanmoins vouloir « en faire des frères et les attirer à nous », afin qu'ils ne s'opposent pas à l'installation du Mandat et à ses « bienfaits ».

13 AMAE-N, BA (1880-1922), Car 102, Note sur l'Union Libanaise.

14 AMAE-N, BA (1880-1922), Car 102, lettre du 23 janvier 1919, BA, la rédaction du Courrier de la Plata au Min. de France à BA.

15 La Sociedad Unión Libanesa, La Unión Libanesa, BA, número extraordinario, 1940.

16 AMAE-N, BA (1880-1922), Car 102, Eco Americano, n 4, 15 de junio de 1921.

17 AMAE-N, BA (1880-1922), Car 102, rapport manuscrit, daté du 31 août 1921, par Checri Abi Saab. 
Cette nouvelle situation politique contraint les religieux maronites du Liban à légitimer leur domination sur le Grand Liban par la construction d'une histoire qui démontre que les Maronites, et eux seuls, ont continuellement pris en charge la défense du Liban, faisant donc coïncider histoire du Liban et histoire des Maronites. Pour eux, le Liban est une terre inviolée par les Arabes. En Argentine, les religieux maronites défendent aussi l'idée que le Libanais est un Maronite, et qu'il n'est donc ni Arabe ni Musulman ${ }^{18}$.

Les répercussions de la déclaration du général Gouraud ne se font pas attendre. En Uruguay, un Club Libanés est fondé par des intellectuels orthodoxes en 1921, puis le Comité Patriotico Libano-Siriano est dissous en 1922 au profit d'une Liga Patriotica Libanesa. Très vite, l'identification hybride «syro-libanaise » cesse d'y être utilisée. En effet, les Libanais y étant beaucoup plus nombreux que les Syriens, les élites libanaises peuvent prétendre représenter l'ensemble des collectivités émigrées originaires de la Grande Syrie devant la société d'accueil tout en valorisant l'identification « libanaise ». La situation inverse se produit en Argentine, où l'augmentation numérique constante d'immigrants syriens et le grand nombre de mariages mixtes empêchent les élites libanaises d'opérer immédiatement une telle séparation.

\section{Les identifications indépendantes de la tutelle française}

Pendant la Première Guerre mondiale, le courant qui s'identifie à l'Empire ottoman est minoritaire chez les collectivités ottomanes en Argentine. Ce sont pour la plupart des Musulmans restés fidèles au Calife, successeur du Prophète et protecteur des lieux saints de l'Islam. Le Musulman Saiffuddin Rahhal défend la politique du gouvernement ottoman dans son journal La Bandera Otomana. Ce courant fonde la Media Luna Otomana, association chargée de lever des fonds et de recruter des volontaires pour participer à la guerre aux côtés de l'Empire ottoman. Toutefois, certains Chrétiens s'identifient aussi à l'Empire ottoman, tel Jorge Sawaya.

Au cours de la seconde moitié du XIXe siècle, une identification arabe apparaît dans la province de Syrie. Tout en acceptant l'identification ottomane, celle-ci se fonde sur une langue et une histoire distinctes des autres groupes ethniques de l'Empire. Cette identification arabe est construite par des Chrétiens qui veulent surmonter les clivages religieux avec les Musulmans en définissant une identité ethnique valable pour tous dans le cadre d'une Syrie autonome (Cloarec et Laurens, 2000 : 18). Elle se retrouve chez les émigrés présents en Argentine. Près de la moitié d'entre eux s'identifient comme Arabes lors du recensement de 1895, tout en assumant pour la plupart leur nationalité ottomane (Jozami, 1993 : 193).

Le nationalisme arabe, qui suppose l'idée d'un projet politique visant la séparation de l'Empire ottoman, n'apparaît qu'avec la Première Guerre mondiale. Face à l'impiété du régime des Jeunes Turcs, le Shérif Hussein de La Mecque organise en 1916 une révolte arabe contre le régime ottoman en se proclamant « Roi des Arabes » et " Commandeurs des croyants ». En octobre 1918, la création du Royaume arabe de Damas dirigé par Fayçal

18 Los maronitas constituyen el pueblo oriental más tradicionalmente católico, El Pueblo, 9 de febrero de 1934. 
constitue la première expérience nationale arabe. Bien que ce royaume soit placé sous l'autorité d'Hussein, Fayçal adopte un discours très tolérant, puisqu'il proclame l'égalité confessionnelle. Le nationalisme arabe de Fayçal s'éloigne de l'arabo-islamisme de son père et prend une teinte plus libérale (Cloarec et Laurens, 2000 : 61). En Argentine, la revendication identitaire arabe ne prend son essor qu'après la Première Guerre mondiale. Les dirigeants syriens et libanais favorables à l'établissement d'un Grand Royaume arabe font alors pression pour que les puissances européennes tiennent leur promesse de former un Grand Royaume arabe. À Buenos Aires, Khalil Sa'adeh, aidé de Felipe Ta'an Homad et de Jorge Sawaya, fonde le Partido Patriótico Arabe en 1919. Il attaque les demandes de Checri Ganem, fer de lance des ambitions françaises en Orient. Quelques mois plus tard, Khalil Sa'adeh part au Brésil, Jorge Sawaya lui succède à la tête du Partido Patriótico Árabe. En mars 1920, la déclaration d'indépendance du Royaume arabe de Damas avec Fayçal comme Roi comble de joie ce parti, qui se considère comme son représentant en Argentine. À Jujuy, à l'occasion du centenaire du général Belgrano, un groupe de Musulmans arbore un drapeau du Royaume arabe de Damas ${ }^{19}$. Très vite, la puissance française réprime ce mouvement d'indépendance en Syrie, ce qui provoque l'indignation des intellectuels concernés en Argentine. En novembre 1920, Jorge Sawaya, au nom du Partido Patriótico Árabe, envoie un télégramme à la Société des Nations « en représentation de 100000 Syro-Arabes en Argentine une assemblée générale de 800 délégués proteste énergiquement devant la Société des Nations contre l'occupation franco-britannique et l'abus en territoires arabes de Syrie, Liban, Palestine et Mésopotamie et réclame l'indépendance absolue et l'évacuation immédiate $»^{20}$. Adhérant à l'arabisme teinté de syrianisme véhiculé par les nationalistes du Royaume arabe de Damas, les leaders de ce mouvement soutiennent donc une identification syro-arabe. Cette dernière disparaît quelques années plus tard face à la prépondérance de la référence identitaire arabe, conformément à l'évolution du nationalisme arabe au Moyen-Orient.

Grâce à ses contacts diplomatiques avec les autorités argentines, la France tente plusieurs fois de faire interdire toute bannière séditieuse arborée par les nationalistes arabes. Leurs premières tentatives rencontrent peu de succès, puisque les drapeaux des États sous Mandat français ne sont pas officialisés et que les droits de la France sur ces pays ne sont pas reconnus internationalement. Mais à partir de 1923, les requêtes françaises sont régulièrement acceptées par les autorités argentines. La France parvient alors à réduire, voire à empêcher l'expression d'une identification arabe devant la société argentine. Jusqu'à l'officialisation du Mandat français sur la Syrie et le Liban (1923), la France codifie donc en partie les diverses identifications des élites ottomanes.

19 AMAE-N, BA (1880-1922), Car 102, lettre du 27 Août 1920, le Consul de France à Rosario, à Mr le Chargé d'Affaires de France à BA.

20 AMAE-N, BA (1880-1922), Car 102, lettre du 14 nov. 1920, BA, Checri Abi Saab à Mr le Min de Fr en Arg. 


\section{LA CONSTRUCTION D'IDENTIFICATIONS ÉMANCIPATRICES DE LA TUTELLE MANDATAIRE (1923-1930)}

Au cours de cette période charnière (1923-1930), la majorité des élites syriennes et libanaises présentes en Argentine s'émancipent de la tutelle mandataire, en construisant des identifications alternatives à celles imposées et contrôlées par la France.

\section{La valorisation d'une identité ethnique arabe par certaines élites anti- mandataires}

Comme le Mandat trouve sa justification dans l'incapacité des peuples à se gouverner eux-mêmes, il n'y a rien d'étonnant à ce que les élites anti-mandataires mettent en valeur leurs capacités civilisatrices, prouvant par là même leur capacité à s'autogérer. Pour s'émanciper, les mouvements anti-colonialistes imaginent généralement une identité culturelle indépendante de leur colonisateur. Le courant de contestation anti-mandataire le plus puissant valorise donc l'identité ethnique arabe, pour s'opposer aux puissances européennes qui ont complètement nié les revendications d'un Grand Royaume arabe. Dans un article de la Gaceta Árabe, Elias Amar l'explicite très bien : « on ne doit pas oublier que notre race [arabe] est en contradiction totale avec les intérêts de la France en Orient $»^{21}$. La majorité des élites musulmanes et orthodoxes valorisent l'identification ethnique arabe alors que seule une minorité d'élites maronites la valorisent, tels Habib Estefano, ancien prêtre maronite ayant participé à la constitution du Royaume arabe de Damas, et Alejandro Schamun. Plusieurs élites s'identifient devant la société argentine comme des « Arabes », tel le Libanais Jorge Sawaya, qui fonde en 1926 le périodique Renovación, vocero de la colectividad árabe. Un an plus tard, il transforme le Partido Patriotica Árabe en Partido Pro-Independencia de los Estados Árabes, et milite désormais pour l'indépendance de chaque pays arabe (Syrie, Liban, Palestine, Transjordanie), avec l'espoir qu'ils se réunissent à l'avenir au sein d'un seul État si telle est la volonté du peuple. L'objectif secondaire est de demander l'indépendance des autres États arabes, afin de former une «Confédération républicaine des États arabes ». À ce moment-là, la Syrie n'est plus le principal foyer d'arabisme, puisque le roi Fayçal dirige la Transjordanie, ce qui explique la volonté de Jorge Sawaya de s'identifier à la fois à la Grande Syrie et à la Transjordanie. Jorge Sawaya est influencé, comme d'autres intellectuels orthodoxes et musulmans, par la doctrine du nationalisme panarabe qui prône la réunion de tous les Arabes - y compris les populations d'Afrique du Nord - sous un même gouvernement. Les élites musulmanes sont les premières à lancer des mots d'ordre de solidarité avec les musulmans d'Afrique du Nord, tels Saifuddin Rahhal, Mohamed Murad et Hossin Ali Hallar. Elles exaltent devant la société argentine les vertus de la race arabe, la contribution des peuples arabes à la civilisation mondiale et notamment l'apport de l'occupation musulmane en Espagne au Moyen Âge.

À la même période, le Maronite Alejandro Schamún met également en valeur les vertus de la race arabe. Il définit la collectivité migrante comme « syrienne », " dénomination qui comprend les originaires du Liban et de la Palestine comme membres ethniques

21 ¿Somos Árabes?, La Gaceta Árabe, BA, 6 de mayo de 1928. 
d'une Syrie unique » (Schamun, 1924 : 7). Bien qu'il se définisse lui-même de «nationalité libanaise » devant le consul de France ${ }^{22}$, cet intellectuel défend un syrianisme englobant Syrie, Liban et Palestine, et basé sur l'homogénéité ethnique arabe de ses habitants. Ses convictions anti-mandataires le motivent sans doute à promouvoir une identification arabe des Syriens, à un moment où la plupart des autres élites libanaises chrétiennes ont cessé d'y adhérer.

Dans un discours tenu devant la société locale argentine, Habib Estefano revendique être « fils de cette terre sainte du Liban et de la Syrie [...], moi, fils des Phéniciens qui ont amené en leurs temps leur civilisation à l'Europe alors barbare, moi représentant des Syriens de Damas et de la civilisation d'Andalousie $»^{23}$. En identifiant les Libanais à leurs ancêtres phéniciens, les Syriens à l'héritage cananéen, Habib Estefano se réfère à la théorie du sémitisme scientifique, qui insiste sur la continuité historique entre l'Orient sémite de l'Antiquité et l'époque contemporaine et qui considère que les Arabes doivent avant tout être définis par leur appartenance aux races sémites (Cloarec et Laurens, 2000 : $62)$.

Les élites qui cherchent leurs référents culturels dans leur « origine » arabe doivent conquérir leur intégration en faisant accepter une culture et une race différente de la race blanche ou latine au sein de la société argentine. Par conséquent, elles se doivent d'affirmer que la contribution de l'immigré arabe au crisol de razas argentin est positive. En 1924, Alejandro Schamún écrit que « le jour où se fera la révision définitive de l'ethnicité nationale [argentine], le Syrien sera sans aucun doute l'élément le plus pur du métal commun » (Schamun, $1924: 11)$.

À la fin des années 1980, lors de conférences très suivies ${ }^{24}$, Habib Estefano transforme cette lutte en lançant l'idée que les Arabes sont des « frères de race » des HispanoAméricains. Dès 1925, pendant son séjour en Espagne, cet intellectuel se fait le promoteur de l'hispanisme arabe-américain, une théorie selon laquelle les conquistadors arrivés au XVIe siècle en Amérique étaient fortement imprégnés de culture et de sang arabe, puisque les villes andalouses du Guadalquivir, têtes de pont de la colonisation, étaient auparavant le siège des Califats et des Empires musulmans (Benumeya, 1955 : 41). Pour cet intellectuel, " le sang des Arabes circule dans les veines des Hispano-Américains » (Estefano, 1931 : 290). Les conquistadors ont aussi apporté du sang "phénicien » en Amérique, puisque les Phéniciens ont fondé les villes de Cadiz et de Malaga. Cela lui permet de considérer que les immigrés libanais, syriens, palestiniens et les Américains contemporains partagent un même patrimoine génétique, une même idiosyncrasie. Ses compatriotes ne représentent donc pas un danger de dénaturation du crisol de razas, mais au contraire apportent du sang « de souche » aux sociétés latino-américaines. Ce discours d'intégration s'inscrit dans un contexte où certaines élites argentines remettent en cause le rôle civilisateur de l'immigrant et revalorisent la figure du criollo, du gaucho et dans une moindre mesure de l'indigène (Devoto, 2003 : 281). Ces dirigeants perçoivent avec bienveillance

22 AMAE-N, BA (1887-1925), Car 99, carta, 30 de septiembre de 1920, BA, Alejandro Schamun al Ministro de Francia en BA.

23 AMAE-N, BA (1923-1945), Car 172, art. Reunión del Instituto popular de conferencias, La

Prensa, 19 de septiembre de 1925.

24 La Gaceta Árabe, 15 de Abril de 1928. 
l'arrivée de collectivités étrangères qui peuvent revendiquer une proximité raciale avec les Argentins, comme les Espagnols, puisqu'ils apportent du sang « de souche » à la nation argentine en formation.

\section{Une nouvelle identification syro-libanaise à tonalité arabe}

Alors que c'est la France qui a encouragé la création d'une identification hybride syro-libanaise pendant la Première Guerre mondiale, il semble bien que celle-ci se consolide par opposition aux autorités mandataires. En 1922, les élites indépendantistes libanaises, les élites nationalistes arabes et indépendantistes syriennes, se rapprochent et s'unissent au sein d'un Comité sirio-libanés pour affronter le même ennemi. Le Druse libanais Felipe Ta'an Homad en est l'instigateur ${ }^{25}$. L'année précédente, il a participé en tant que délégué des collectivités syriennes d'Argentine au congrès syro-palestinien à Genève. Sous la présidence de l'Émir Michel Lutfallah, ce congrès avait rassemblé en août 1921 tous les émigrés opposés au Mandat français sur la Syrie, généralement d'anciens partisans d'une Grande Syrie unitaire. Les Palestiniens présents au Congrès refusent toutefois l'inclusion de la Palestine au sein de la Syrie et imposent l'ajout du terme «palestinien » à la nouvelle organisation. De retour en Argentine, Ta'an Homad mobilise ses compatriotes pour accuser les autorités françaises de cacher les recommandations de la Commission King-Crane. Cette commission avait procédé en 1919 à une consultation des populations syriennes et les résultats étaient éloquents : elles n'acceptent ni un Mandat français ou anglais, ni l'implantation d'un État juif, mais réclament leur indépendance.

Outre Felipe Homad, le docteur Jorge Sawaya, les frères Moises et Salomon Busader, Massud Posleman, Wadik et Alexandre Schamun, Husni Abdul Malik, Esbir Gorayeb, Constantin Melhem, Elias Amar, et Moisés José Azize sont les principaux leaders de ce Comité sirio-libanés. Ils organisent une manifestation publique le 23 juillet 1922 à Buenos Aires contre le Mandat français en Syrie et au Liban et contre l'implantation d'un Foyer juif en Palestine. Ces élites anti-mandataires, tant libanaises que syriennes, se sentent plus unies par leur combat contre l'occupant que divisées par les prétentions politiques distinctes des nationalismes auxquels elles se réfèrent.

Au milieu des années 1920, une élite syrienne et libanaise anti-mandataire se forme autour de deux commerçants fortunés, Moisés José Azize et Moisés Busader. Disposant de moyens économiques importants, cette élite fonde des institutions qualifiées de syro-libanaises et auto-proclamées représentatives : El Banco Sirio-libanés del Rio de la Plata en 1925, puis la Camara de Comercio Siriolibanesa, le Patronato Sirio-libanés de Protección al Inmigrante en 1928, et le Diario Siriolibanés en 1929. En utilisant cette identité hybride, ces responsables tentent de persuader leurs compatriotes originaires de la Grande Syrie qu'ils constituent une seule et même collectivité. Du fait de l'existence d'un courant libaniste en son sein, la faction syrienne de cette élite dirigeante n'ose pas appeler cette collectivité sirios. Néanmoins, Moisés José Azize interprète le contenu de l'identité hybride syro-libanaise de façon à gommer les particularités de l'identité libanaise. Avec certains de ses amis, il considère que cette identité hybride conforte l'idée d'une Grande

25 AMAE, BA (1923-1945), Car 170, rapport manuscrit, daté du 20 juillet 1922, remis par Checri Abi Saab au consulat de France à Buenos Aires. 
Syrie indépendante de la France, dotée d'une composante ethnique arabe. En 1929, le Diario Siriolibanés recommande l'emploi du qualificatif syro-libanais, car Syriens et Libanais partagent la même idiosyncrasie, la même langue et sont soumis à la même nation $^{26}$. En juillet 1936, lors de l'inauguration de la succursale du Banco Siriolibanés del Rio de la Plata à Tucumán, Moisés José Azize se présente comme « nosotros los Árabes " dans son discours ${ }^{27}$. Néanmoins, le Diario siriolibanés évite toute référence à l'anti-sionisme. Cela s'explique par le fait que plusieurs Syriens de religion juive (José Jorge, Nissim Teubal) appartiennent à cette élite qui dirige les institutions syro-libanaises (Klich, 1995). Jusqu'à la fin des années 1930, ceux-ci sont peu attirés par le sionisme politique, et adhèrent à une identification syro-libanaise à tonalité arabe.

Cette interprétation de l'identification syro-libanaise suscite parfois l'opposition de certains alliés. Moisés José Azize est souvent contraint d'accepter des compromis politiques : le local de la succursale du Banco Siriolibanés à Tucumán est par exemple orné de drapeaux argentin, libanais et syrien ${ }^{28}$. Dans d'autres cas, des tensions existent au sein de cette élite, au point de refuser qu'un quelconque drapeau autre que l'argentin soit hissé dans la salle du conseil d'administration. Cela laisse donc à penser que les principaux objectifs de cette union sont à la fois économiques pour les élites et sociaux pour la collectivité, mais ne sont pas politiques.

La diffusion du Díario Siriolibanés et les nombreux voyages de Moisés José Azize à l'intérieur du pays encouragent la diffusion du terme sirio-libanés, qui se généralise dans les institutions de tout le pays dans les années 1930. Elias Amar, directeur de La Gaceta Arabe, emploie aussi le terme siriolibaneses pour désigner ses compatriotes. Il mentionne ainsi « les siriolibaneses, qui représentent si dignement la race arabe en Argentine ${ }^{29}$. De plus, Jorge Sawaya change le nom de son journal, de vocero de la colectividad árabe pour vocero de la colectividad sirio-libanesa en 1929 (Jozami, 1993 : 199). L'identification nationale arabe s'estompe donc au profit d'une identité syro-libanaise désormais consolidée, dans la mesure où cette dernière s'appuie sur une composante ethnique arabe.

Pendant cette période, l'identification syro-libanaise se construit donc majoritairement à partir d'une composante arabe, mais il faut aussi en souligner la plasticité, puisque certains chrétiens libanais la soutiennent avec une autre connotation.

\section{La réponse libanaise : une identification syro-libanaise à tonalité « orientale »}

Qu'elles soient anti-mandataires ou pro-mandataires, les élites libanaises favorables à un Liban indépendant ne s'identifient pas systématiquement comme Libanais devant la société d'accueil. L'utilité d'une union entre Syriens et Libanais, l'importance

26 Lo que significa el ambiente siriolibanés en la Argentina, Diario Siriolibanés, BA, 9 de agosto de 1930. Cité par Jozami, 1993, p. 199.

27 El Eco de Oriente, Tucumán, número extraordinario, 25 de Julio de 1936.

28 El Eco de Oriente, Tucumán, número extraordinario, 25 de Julio de 1936.

29 La Gaceta Árabe, Buenos Aires, 4 de marzo de 1928. 
de la collectivité syrienne et des mariages mixtes, ou la volonté de ne pas offenser les amis syriens, conduisent nombre d'entre eux à accepter le terme hybride « syro-libanais » pour désigner leurs compatriotes. Ainsi, la Sociedad Damas de Misericordia fondée en 1916 à Buenos Aires sous les auspices du père maronite Elias Gorayeb, et composée essentiellement de femmes originaires du Mont-Liban, change de nom en 1923 au profit de Asociación de Beneficiencia Sirio-Libanesa. En 1925, cette association se transforme en Asociación Pro-Hospital Sirio-Libanés.

Pour conserver une solidarité entre tous les immigrés originaires de la Grande Syrie, tout en évitant de recourir à une identification ethnique arabe, les Libanais chrétiens reprennent l'idée d'une identification orientale qui leur avait été attribuée par les Européens depuis le XIXe siècle. Dans la littérature orientaliste, les intellectuels européens prétendent dévoiler le passé antique grandiose de l'Orient et diffusent une image exotique des « Orientaux ». Néanmoins, ces ouvrages opposent de façon binaire l'Occident et l'Orient, ce qui aboutit logiquement à la supériorité de l'un sur l'autre (Saïd, 1997). En Argentine, les élites libanaises chrétiennes reprennent cette identification orientale pour maintenir la cohésion au sein de ce qu'elles considèrent comme un « conglomérat racial ».

Bien que les élites maronites s'enorgueillissent d'être les descendants des Phéniciens devant leurs compatriotes libanais, elles ne peuvent défendre une telle identification lorsqu'elles s'adressent à l'ensemble des collectivités syriennes et libanaises. Dans un discours public, le Maronite Checri Abi Saab entretient le flou sur l'identité ethnique des migrants, mentionnant son appartenance à « une race qui fait du devoir un culte », sans définir davantage cette « race ». Il se réfugie dans l'expression poétique des paysages de l'Orient pour mettre en valeur les qualités des migrants, afin de ne pas avoir à affronter l'épineuse question de la différentiation d'identité (et donc de race) entre les Libanais et les Syriens ${ }^{30}$.

De même, en 1935-1936, un conflit au sein des collectivités syriennes et libanaises de Tucumán vient confirmer cette volonté chrétienne de valoriser l'identification à la culture « orientale ». La commission d'hommage au Centenaire de l'Argentine, qui est chargée depuis plus d'une vingtaine d'années d'offrir un monument de la part des collectivités syriennes et libanaises, projette d'élever un minaret. Les Chrétiens libanais rejettent formellement cette idée : un éditorial du journal El Eco de Oriente, dirigé par le Melkite Nagib Baaclini, considère que le projet manque de symbolisme « pour que ce soit un cadeau spécifiquement oriental sans qu'il puisse comporter aucune intention de représenter les spécificités d'une religion ou d'une autre $\rangle^{31}$. Les caractéristiques du monument doivent selon eux être représentatives « de l'art et de la glorieuse civilisation orientale $»^{32}$.

Les religieux maronites ont une plus grande liberté dans leurs discours publics et insistent d'abord sur l'intégration de leur collectivité maronite-libanaise. Dans ce but,

30 AMAE-N, BA (1923-1945), Car 170 bis, discours prononcé par Checri Abi Saab pendant la cérémonie en l'honneur du Docteur Julio A. Mendez le 23 février 1927, publié dans la Gaceta Arabe, le 27/2/1927.

31 La Comisión de Homenaje al Centenario debe trabajar, El Eco de Oriente, Tucumán, 14 de noviembre de 1936.

32 El grave asunto del Monumento, El Eco de Oriente, Tucumán, 11 de mayo de 1935. 
ils adoptent une stratégie de mimétisme avec la société locale, en affirmant que «les Maronites sont les Catholiques apostoliques romains de l'Orient, comme les latins sont les Catholiques de l'Occident $»^{33}$. Par souci de favoriser l'intégration de l'ensemble des émigrés originaires de la Grande Syrie, les religieux maronites leur attribuent collectivement l'identification orientale, tout en distinguant clairement le Libanais du Syrien. En 1927, devant un public argentin, le père Elias M. Gorayeb déclare qu'il faut les appeler Orientaux, puis les subdiviser en Orientaux syriens, Orientaux libanais, etc. ${ }^{34}$ Toutefois, l'emploi de cette dénomination est très limité, car une confusion peut surgir avec les citoyens de la Republica Oriental del Uruguay, généralement appelés Orientales dans les pays du Cône Sud.

\section{Refuser la catégorisation « de papier » organisée par la France}

Après la mise en place du Mandat français sur la Syrie et le Liban, les élites anti-mandataires sont très vite confrontées à un événement hautement symbolique : c'est la France, leur puissance tutélaire, qui est chargée d'organiser la collecte des options de nationalité des émigrés originaires de la Grande Syrie. Juridiquement titulaires jusqu'alors de la nationalité turque, les chefs de famille doivent poser une option auprès du consulat de France avant le 30 août 1926 s'ils veulent acquérir la nationalité libanaise ou syrienne. Mais dès qu'elles sont au courant de la procédure d'option, c'est-à-dire à partir du milieu de l'année 1925, la plupart des élites syriennes et libanaises en Argentine s'opposent ouvertement à l'option. Cette opposition, menée par Moisés Busader, conteste le fait que ce soit le gouvernement français qui valide l'option et non pas le Liban ni la Syrie. En critiquant l'option, ces intellectuels interrogent l'essence même du Mandat, c'est-à-dire la différence entre le devoir de la France de mener ces pays à l'indépendance et la réalité du pouvoir politique contrôlé par les autorités mandataires (Brégain, 2008 : 68). Conscients des conséquences de l'absence d'option, mais certains que le droit est de leur côté, ils poussent leurs compatriotes à ne pas opter, car c'est inutile. Ils sont en effet convaincus que les gouvernements libanais et syrien prendront des mesures pour les confirmer dans la nationalité de leur pays d'origine. Certaines élites, minoritaires, militent toutefois pour que leurs compatriotes optent pour une nationalité ou l'autre : c'est le cas de Checri Abi Saab, alors drogman au consulat de France à Buenos Aires, et des religieux maronites. La propagande des élites anti-mandataires atteint son objectif : seuls 4101 émigrés optent pour l'une des nationalités libanaise ou syrienne, pour eux et leur famille ${ }^{35}$. Dès lors, les administrations françaises considèrent que les émigrés non-optants sont de nationalité turque.

Conscients de l'enjeu que représentent les mentions figurant sur les papiers d'identité, les dirigeants syriens et libanais éparpillés dans les terres d'émigration luttent contre la catégorisation réalisée par les autorités mandataires, qui leur attribuent la nationalité turque par défaut d'adhésion à l'option de nationalité. Pour cette raison, en 1927, le ministre des Affaires étrangères français décide que ne soit pas mentionnée sur les passeports la nationalité turque des émigrés non-optants, ce qui serait susceptible de les blesser,

33 Informe de la Asociación Protectora del Colegio San Marón, op. cit, p. 3.

34 Ibid., pp. 53-54.

35 AMAE-N, BA (1923-1945), Car 169 et 173. 
mais par contre d'apposer la mention « passeport pour personne d'origine syrienne (ou d'origine libanaise) $»^{36}$. Toutefois, l'administration française ordonne aux agents consulaires de limiter aux cas exceptionnels la protection accordée aux émigrés non-optants, ce qui les marginalise juridiquement.

Pendant plusieurs années, les élites libanaises anti-mandataires conservent l'espoir de retrouver rapidement leur nationalité, mais leurs premières tentatives échouent. À Buenos Aires, la Unión Libanense organise un congrès le 5 septembre 1930 « dans le but de trouver la meilleure solution pour régulariser la situation de la majorité de nos compatriotes libanais en ce qui concerne leurs droits politiques et civils dans leur pays natal $\aleph^{37}$. L'objectif de ce congrès est aussi d'unir les Libanais au sein d'une entité qui assurerait leur représentation diplomatique devant la société d'accueil. Toutefois, la révolution du 6 septembre et le décret du général Uriburu interdisant les réunions mettent un terme au projet ${ }^{38}$.

En Argentine, pour éviter d'avoir recours aux administrations françaises, certaines élites anti-mandataires tentent d'obtenir le droit de délivrer aux immigrés non-optants des papiers d'identité valables auprès de la police. Dès 1926, Habib Estefano convainc les autorités policières de Mendoza de délivrer des pièces d'identité aux immigrés d'origine syrienne ou libanaise « non plus sur présentation du certificat de nationalité que délivrent les postes consulaires, mais sur la simple vue de la carte de membre d'une association $»^{39}$. $\mathrm{Au}$ cours de la même année, lors d'une conférence, Habib Estefano propose de constituer un « cercle syro-libanais », qui s'occuperait de défendre leurs intérêts et de délivrer des cartes d'identité, sans avoir recours au ministre de France ${ }^{40}$. Faute de moyens financiers, il ne peut pas faire aboutir son projet tout seul. Finalement, Moisés José Azize et Moisés Busader récupèrent cette idée et la mènent à son terme en fondant, en mars 1928, le Patronato Sirio Libanés, qui obtient la capacité d'émettre des certificats d'identité reconnus par les autorités locales.

Afin de résoudre leur situation juridique qui reste indéfinie et pour ne plus dépendre des autorités françaises lorsqu'ils veulent obtenir des papiers d'identité, certains leaders anti-mandataires se font naturaliser argentins et encouragent leurs compatriotes à faire de même. En Argentine, Habib Estefano demande sa naturalisation à la fin de l'année 1927, Moisés José Azize, Mécia Dantur en 1928, Massud Posleman en 1929, Alejandro Schamun en 1932. Suite à la propagande que ces élites diffusent dans leurs journaux, une grande vague de naturalisation a lieu entre 1927 et 1931, avec 211 naturalisations d'immigrés d'origine syrienne et libanaise en 1927, 278 en 1928, 418 en 1929, 439 en 1930 et 234 en $1931^{41}$. Après 1931, environ cinquante naturalisations sont recensées chaque année.

36 AMAE-N, BA (1923-1945), Car 169, lettre n 115, le 21 mai 1927, Paris, le MAE à Mrs les ADC de France à l'étranger.

37 La Libertad, Tucumán, 2 de septiembre de 1930.

38 La Sociedad Unión Libanesa, La Unión Libanesa, BA, número extraordinario, 1940.

39 AMAE-N, BA (1923-1945), Car 173, lettre n 40, le 5 juin 1926, Mendoza, Jacques Pingaud au Min de France à BA.

40 AMAE-N, BA (1923-1945), Car 170, rapport du 30 mai 1926, de Checri Abi Saab, intitulé « Réunion Syriolibanaise ».

41 Archivo de la cámara nacional electoral de Buenos Aires, Registro de Cartas de Ciudadanía 1927-1944. 
Pour les intellectuels, cet acte de naturalisation révèle probablement leur patriotisme à l'égard de la patrie d'origine, dans le sens où ils prouvent leur indépendance de la tutelle française.

La procédure d'option organisée et contrôlée par la France, influe sur l'identification des élites émigrées, puisqu'elle engendre une réaction d'émancipation de la tutelle française, et une volonté de procéder elles-mêmes à la " catégorisation de papier ». Cependant, la procédure d'option modifie peu l'identification des élites par rapport à la société argentine.

Pendant les années 1920, les élites s'émancipent de la tutelle mandataire en valorisant l'identité ethnique arabe, en réinterprétant le contenu de l'identification syrolibanaise et en refusant la catégorisation de papier opérée par la puissance mandataire. Comme ils doivent leur identité à leur propre mobilisation collective, les émigrés syriens et libanais méritent le titre de groupe et non celui de catégorie administrative (Noiriel, $1991: 319-320)$.

\section{LA (RE)NAISSANCE DES IDENTIFICATIONS LIBANAISE ET ARABE (1930-1946)}

Après 1930, la France n'exerce plus de réelle influence sur les affirmations identitaires des élites syriennes et libanaises émigrées. Cette période se caractérise par la promotion active des identifications libanaise et arabe devant la société argentine. L'identification strictement syrienne n'est revendiquée que de façon très ponctuelle, comme en 1936, lorsque la menace de l'indépendance d'Alexandrette pousse certains émigrés à se rassembler pour défendre l'intégrité territoriale de leur nation ${ }^{42}$. Toutefois, elle est invoquée de manière plus systématique à partir de 1939 par Antun Saadeh, président du Parti Populaire Syrien, résidant alors en Argentine. Néanmoins, s'il réussit à promouvoir ses idées auprès de certaines familles syriennes, il éprouve de grandes difficultés à rallier les principaux intellectuels syriens et libanais.

\section{La défense d'un Liban indépendant de la Syrie}

Dès la fin des années 1920, les intellectuels libanais des capitales provinciales argentines reconstruisent une identification libanaise distincte de l'identification syrienne, avec la création du Centro Libanés à Mendoza (1928) et du Centro Libanés à Tucumán (1931). La France les seconde parfois dans cette tâche dans le but de s'assurer leur fidélité. Ainsi, Émile Gissot, consul de France à Mendoza, parvient à rassembler les principales associations libanaises des villes de province pour qu'elles célèbrent leur indépendance le $1^{\text {er }}$ septembre 1933, alors que certaines d'entre elles étaient clairement opposées au Mandat (Brégain, 2008 : 226).

42 AMAE-N, BA (1923-1945), Car 171, lettre du 28 déc. 1936, le secrétaire général de la Ligua de la Juventud Siria à l'Amb. de France. 
Dans la capitale argentine, l'ensemble des élites libanaises s'identifient à un Liban indépendant de la Syrie à partir de 1936. Cette année-là, au mois de mars, les dirigeants musulmans du Liban, ainsi que quelques dirigeants chrétiens, acceptent que certaines régions du Liban soient annexées à la Syrie. Rapidement, les tensions augmentent dans les rues de Beyrouth : les Musulmans déclarent que le Liban est un pays arabe, et qu'il doit par conséquent être rattaché aux pays arabes, tandis que les Chrétiens invoquent l'origine phénicienne du Liban pour justifier sa non-arabité (Kaufman, 2004 : 128). Le Patriarche maronite encourage alors la création d'un Front national libanais, dirigé par Yusuf al-Saouda, l'un des leaders de l'identification phénicienne du Liban. En juillet 1936, une délégation syrienne se présente à Paris pour réclamer l'annexion du Liban ou tout au moins de quelques-unes de ses plus importantes villes maritimes. Lorsqu'ils l'apprennent, les intellectuels maronites en Argentine réagissent immédiatement.

Le 15 août 1936, le père maronite Miguel Latuf Inderi rassemble plus d'un millier d'émigrés libanais au Colegio San Marón de Buenos Aires. Il les invite alors à s'unir pour protéger l'indépendance et l'intégrité du Grand Liban (Libro Purpura, 1996 : 27). Le 28 août 1936, une délégation libanaise menée par le président de l'Unión Libanesa, Domingo Kairuz, et le père supérieur maronite, se présente à l'ambassade de France pour remettre une lettre de protestation « contre les affirmations de la délégation syrienne ${ }^{43}$. La délégation « tient à [y] remercier le gouvernement français de l'attitude qu'il a adoptée pour défendre l'intégrité du territoire libanais ». Le 18 octobre 1936, la plupart des élites libanaises chrétiennes, toutes tendances politiques confondues, réunies au Colegio San Marón, fondent l'Asociación Patriótica Libanesa (APL). La promesse de la signature prochaine d'un traité franco-libanais incite la plupart des intellectuels libanais chrétiens anti-mandataires à rejoindre leurs compatriotes jusque récemment favorables au Mandat, autour du projet d'intégrité et d'indépendance future du Grand Liban. Au sein de l'APL se côtoient ainsi d'anciens membres de l'Alliance libanaise (José Juairy, José Curi, Miguel Sfeir, José Akel, etc.) et certains de leurs anciens opposants politiques (Alejandro Schamun, Moisés Busader, Domingo Kairuz, Rachid Rustom). Alejandro Schamun, qui jusqu'au début des années 1930 prônait la constitution d'une Grande Syrie incluant le Liban ${ }^{44}$, rallie aussi l'idée d'un Liban indépendant. Toutefois, plusieurs élites libanaises orthodoxes sont réticentes à cette initiative et les Musulmans, comme Mohammed Murad, et les Druses tels l'Émir Emin Arslan et Felipe Ta'an Homad, militent clairement contre.

Bien que menée avec diplomatie et respect, cette stratégie de séparation des collectivités syriennes suscite des réticences de la part de certaines élites, qui ont peur d'y perdre des avantages économiques, politiques ou électoraux. En dépit des ces enjeux, les élites libanaises chrétiennes ne peuvent que s'incliner devant les impératifs politiques.

Pour la société argentine, la création de l'APL marque la présentation officielle d'une identité libanaise distincte de l'identité syrienne, basée sur l'existence d'une « différence ethnique indiscutable au sein du conglomérat racial dans lequel la collectivité

43 AMAE-N, BA (1923-1945), Car 173, lettre du 28 août 1936, Mr Frederic Knobel, chargé d’Affaires de France en Argentine à Mr Yvon Delbos MAE.

44 Schamun, La Siria, La Nación, 1930, non daté précisément (AMAE-N, consulat de France à BA, Car 99, Dossier 1530). 
développe ses activités $»^{45}$. José Juairy, rédacteur de l'Azzaman et connu pour ses déclarations sur l'identification des Libanais à leurs ancêtres phéniciens, prononce un discours lors de la création de l'association. À partir de ce moment-là, de plus en plus d'élites adhèrent à cette identification phénicienne. D'ailleurs, celle-ci est utilisée pour légitimer la présence des Libanais sur les terres argentines. En 1940, le Maronite Victor Marun affirme que « 17 siècles avant Colomb, les Phéniciens avaient occupé les côtes du Brésil et s'étaient déployés à l'ouest jusqu'au Pérou et au nord jusqu'à Mexico ${ }^{46}$. Il reprend ainsi une thèse largement développée depuis la fin du XIXe siècle par des élites libanaises émigrées dans les pays latino-américains, et notamment au Mexique et au Brésil (Kaufman, $2004:$ 103).

Il semble que l'union des chrétiens libanais au sein de l'Asociación Patriotica Libanesa se construise autour de l'indépendance vis-à-vis de la tutelle française. Le fait que Checri Abi Saab soit absent au moment de la création de cette association reflète l'esprit qui a guidé ses principaux fondateurs. En effet, l'association a clairement pour ambition de représenter diplomatiquement les Libanais à l'étranger, ce qui entraîne symboliquement la perte des fonctions de drogman au consulat de France, poste qu'occupe Checri Abi Saab. Quelque temps plus tard, l'APL prouve son indépendance vis-à-vis de l'Ambassade de France en organisant elle-même la nouvelle procédure de droit d'option de 1938-1939. En 1939, l'APL recueille au moins $80 \%$ des 11453 options libanaises souscrites ${ }^{47}$.

Bien que cette identification à un Liban indépendant soit surtout le fait des Chrétiens, l'épisode patriotique de 1936 se fait officiellement sans cristallisation autour de la religion. Pourtant, si les élites libanaises chrétiennes sont effectivement capables d'accueillir des Musulmans au sein de l'association, c'est parce qu'ils considèrent que leur religion est supérieure aux autres. En 1940, la Unión Libanesa appelle les Libanais des confessions juives et musulmanes à les rejoindre, car un individu ne choisit pas le milieu religieux dans lequel il naît ${ }^{48}$.

Le processus de construction de l'identité libanaise ne s'achève réellement qu'après la Seconde Guerre mondiale, avec l'indépendance effective de la Syrie et du Liban. Alejandro Schamun déclare alors que « certains, en se référant à la nationalité de l'un d'entre nous, disent : c'est un Syrolibanais. C'est une erreur que beaucoup de nos compatriotes commettent eux-mêmes. [...] Individuellement, un homme est soit Syrien, soit Libanais ${ }^{49}$. Les élites libanaises s'aperçoivent par la suite que l'identification siriolibanés peut-être utilisée de manière idéologique par les élites favorables à une Syrie incluant le Liban. Employer ce terme revient finalement à ne pas reconnaître au Liban son état d'indépendance vis-à-vis de la Syrie.

45 Ha quedado constituida en el país la Asociación Patriótica Libanesa con sede en la Capital de la Nación, El Eco de Oriente, Tucumán, 24 de octubre de 1936.

46 Marun, Glorioso Líbano, La Unión Libanesa, BA, número extraordinario, 1940.

47 AMAE-N, Mandat Syrie Liban, $3^{\mathrm{e}}$ versement, Car 96, 97, 112 à 133.

48 La Unión Libanesa, BA, número extraordinario, 1940.

49 Schamun, Quienes somos los residentes de habla árabe, Revista Mensual Siria y Líbano, Asunción, $\mathrm{n}^{\circ}$ 28, febrero de 1949. 


\section{Le réveil d'une identification nationale arabe à connotation islamique}

Dès les années 1920, l'Asociación Pan-Islamismo de Buenos Aires dirigée par Mohammed Murad défend le panarabisme à tonalité islamique diffusé par Chekib Arslan. Cette identification arabe progresse nettement en 1929. Cette année-là, les tensions arabosionistes s'exacerbent en Palestine, du fait de la revendication juive sur le site du Mur des Lamentations à Jérusalem, ce qui est perçu par les Musulmans comme le premier pas vers une appropriation totale des lieux saints musulmans. À l'image d'autres associations « arabes » ou « islamiques », l'Asociación Pan-islamismo de Buenos Aires envoie au début du mois de septembre un télégramme à la Société des Nations, pour protester contre l'imposition du Foyer Juif et du Mandat anglais ${ }^{50}$. Cette identification arabe à connotation islamique ne cesse de se construire tout au long des années 1930, grâce à l'activisme de l'Égyptien Saifuddin Rahhal dans le journal Al Fitra, et à celui de l'Émir Arslan dans $\mathrm{Al}$ Istiklal, du nom du premier parti politique pan-arabe fondé à Jérusalem en 1931.

Sur la proposition de l'Émir Chekib Arslan, un congrès pan-arabe est organisé à Buenos Aires en avril 1941. Ce congrès, présidé par l'Émir Emin Arslan, ambitionne de formuler les vœux de tous les Arabes libres en Amérique. Des délégués d'une dizaine d'associations d'Argentine, dont Hossein Ali Hallar et Mohamed Murad, y participent. La quasi-totalité des délégués sont Musulmans, sauf Rachid Rustom, seul Chrétien présent. Bien que l'ouverture d'esprit du mouvement arabe soit mise en avant, une résolution votée prévoit que « les Arabes ne reconnaissent et ne reconnaîtront pas sur leurs territoires l'existence de minorités religieuses, territoriales ou raciales $»^{51}$. Lors du Congrès ont lieu des manifestations de solidarité avec le dirigeant irakien Rachid Ali, Ibn Saud, et avec les chefs arabes prisonniers Abd el krim et Ali el Taher. Finalement, le Congrès affirme que « la Syrie, y compris le Liban, la Palestine et la Transjordanie forment une unité indivisible » et que les habitants de ces pays réclament l'indépendance. Une commission permanente du premier congrès arabe en Amérique est ensuite instituée et continue à promouvoir l'identification arabe à tendance islamique.

\section{CONCLUSION}

Si en 1915, les autorités françaises soutiennent certaines élites soucieuses d'affirmer leur identité libanaise, elles tentent dès 1917 de leur imposer une identification syro-libanaise, à teneur non arabe, afin de soutenir la politique française d'implantation au Levant. Dans les années 1920, l'élite syrienne et libanaise groupée autour de Moisés José Azize réinterprète cette identité hybride afin d'y intégrer la composante ethnique arabe. Grâce aux contacts politiques tissés par Moisés José Azize, plusieurs responsables politiques argentins reconnaissent les bienfaits de cette immigration siriolibanesa à composante ethnique arabe, ce qui permet d'améliorer le prestige social de « la collectivité » émigrée.

50 AMAE-N, BA (1923-1945), Car 170 bis, note du 5/9/1929, de Checri Abi Saab, intitulée « Les évènements de Palestine ».

51 AMAE-N, BA (1923-1945), Car 171, note de Victor Abi Saab sur le Congrès pan-arabe, non datée. 
À la fin des années 1930, la plupart des élites libanaises chrétiennes présentes en Argentine se rassemblent pour défendre un Liban indépendant de la Syrie. Contrairement à leurs compatriotes présents en Uruguay, elles ne parviennent pas à convaincre la société argentine de ne plus utiliser le qualificatif syro-libanais, ni à lui démontrer leur " origine» ethnique phénicienne. Par la suite, les religieux maronites continuent de refuser l'hypothèse d'une identité arabe du Liban et ce, même si la signature du pacte national de 1943 lie officiellement l'identité libanaise à une origine ethnique arabe.

Après la Seconde Guerre mondiale, les nouvelles générations d'élites abandonnent peu à peu le qualificatif syro-libanais. Du fait de la naissance de la Ligue des États arabes en 1945, de la naissance d'Israël en 1948 et du conflit israélo-palestinien, celles-ci s'identifient de plus en plus souvent comme Arabes.

\section{Références bibliographiques}

BENUMEYA Gil (1955) Sobre las líneas generales de las relaciones hispano-árabes en su evolución actual, Cuaderno de Estudios Africanos, 32, Octubre-diciembre, pp. 39-48.

BESTENE Jorge Omar (1994) Realidad y estereotipos: los « Turcos » en el teatro argentino, Estudios Migratorios Latino-americanos, 9 (26), pp. 143-164.

BREGAIN Gildas (2008) Syriens et Libanais d'Amérique du Sud (1918-1945), Paris, L'Harmattan, $320 \mathrm{p}$.

SELUJA CECIN Antonio (1989) Los Libaneses en el Uruguay, Montevideo, Ed. autor, 224 p.

COOPER Frederick (2005) Colonialism in question. Theory, Knowledge, History, Berkeley, University of California Press, $328 \mathrm{p}$.

CLOAREC Vincent et LAURENS Henry (2000) Le Moyen-Orient au XXe siècle, Paris, Armand Colin, $255 \mathrm{p}$.

CLUB LIBANES de Buenos Aires (1996) Libro Purpura de los Sesenta años del Club Libanés de Buenos Aires, 1936-18 Octubre 1996, Buenos Aires, Valdez, 177 p.

DEVOTO Fernando (2003) Historia de la inmigración en la Argentina, Buenos Aires, Agencia Española de Cooperación Internacional, 528 p.

ESTEFANO Habib (1931) Los pueblos hispano-americanos, su presente y su porvenir, Méjico, ed. culturales, $295 \mathrm{p}$.

HOURANI Albert et SHEHADI Nadim (Dir.) (1992) The Lebanese in the world: a century of emigration, London, Centre for Lebanese Studies and I. B. Tauris, $742 \mathrm{p}$.

JOZAMI Gladys (1993) La identidad nacional de los llamados turcos en la Argentina, Temas de África y Asia, 2, Facultad de Filosofía y Letras, Univ. Buenos Aires, pp. 189-204.

KAUFMAN Asher (2004) Reviving Phoenicia. The search for identity in Lebanon, London, IB Tauris, $277 \mathrm{p}$.

KLICH Ignacio (1992) Criollos and Arabics Speakers in Argentina: An uneasy Pas de Deux, 18881914, in Albert Hourani et Nadim Shehadi Dir., The Lebanese in the world: a century of emigration, London, Centre for Lebanese Studies and I. B. Tauris, pp. 247-284.

KLICH Ignacio (1995) Árabes, judíos y árabes judíos en la Argentina de la primera mitad del novecientos, Estudios Interdisciplinarios de América Latina y el Caribe, 6 (2), pp. 109-143.

KLICH Ignacio (Dir.) (2006) Árabes y judios en América Latina: historia, representaciones y desafios, Buenos Aires, Siglo XXI Editora Iberoamericana, 409 p.

KLICH Ignacio and LESSER Jeffrey (1996) Introduction: «Turco » immigrants in Latin America, The Americas, Washington, 53 (1), July, pp. 1-14. 
MARTOS Sofia (2007) The Balancing Act: Ethnicity, Commerce, and Politics among Syrian and Lebanese Immigrants in Argentina, 1890-1955, PHD in History, University of California, Los Angeles, $456 \mathrm{p}$.

NANCY Michel (Dir.) (1998) Les Arabes du Levant en Argentine, Aix en Provence, IREMAM, $141 \mathrm{p}$.

NOIRIEL Gérard (1991) Réfugiés et sans papiers : La République face au droit d'asile $X I X^{e}-X X^{e}$ siècle, Paris, Calmann Levy, $355 \mathrm{p}$.

REIN Raanan (Dir.) (2008) Árabes y judios en Iberoamérica: similitudes, diferencias y tensiones, Sevilla, Fundación Tres Culturas del Mediterráneo, 460 p.

SAID Edward Wadie (1997) L'orientalisme : l'Orient créé par l'Occident, Paris, Seuil, 422 p.

SCHAMUN Alejandro (1917) La Siria Nueva. Obra Histórica, estadistica y comercial de la colectividad sirio otomana de la Republica Argentina y Uruguay, Buenos Aires, Ed. de Assalam, 236 p.

SCHAMUN Alejandro (1924) Guía Assalam de comercio sirio-libanés en la Republica Argentina, Buenos Aires, Ed. de Assalam, 302 p.

SENKMAN Leonardo (2008), Identidad y asociacionismo de sirios, libaneses y " jalabes » en Argentina, in REIN Raanan Ed., Árabes y judios en Iberoamérica: similitudes, diferencias y tensiones, Sevilla, Fundación Tres Culturas del Mediterráneo, pp. 181-221. 